and disaccharides) and the decrease of dietary fiber as shown in the multivariable analyses (Figure 1). This effect was independent of age, sex, smoker status and BMI.

Conclusion: A higher intake of carbohydrates and a higher consumption of free sugars are associated with higher disease activity in patients with AS.
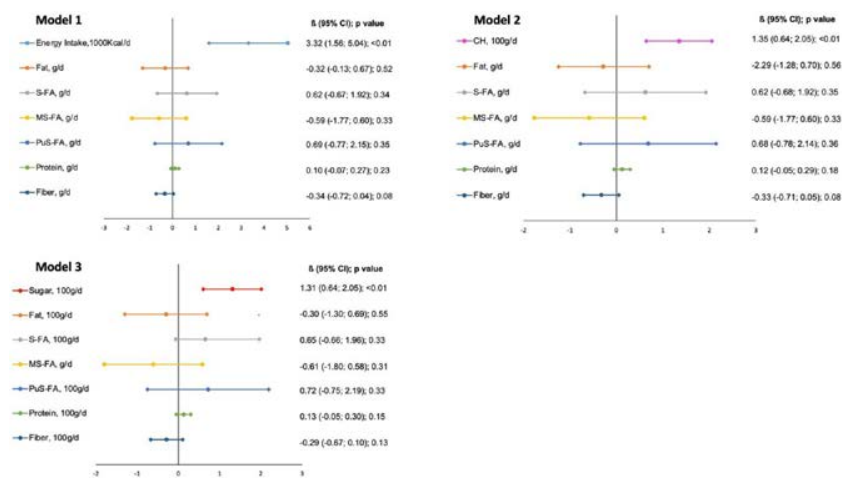

Figure 1. Multivariable linear regression analysis of the association between CRP and nutritional parameters in patients with radiographic axial SpA $(n=104)$, adjusted for age, sex, body mass index and smoker status. Model 1 included variable of total energy intake, model 2 included variable of total intake of carbohydrates $(\mathrm{CH})$ and model 3 included variable of freesugar (monosaccharides and disaccharides).

$\mathrm{B}$, linear regression coefficient; $\mathrm{CH}$, carbohydrates; $\mathrm{Cl}$, confidence interval; MS-FA, monosaturated fatty acids; PuS-FA, polyunsaturated fatty acids, S-FA, saturated fatty acids.

Disclosure of Interests: None declared.

DOI: 10.1136/annrheumdis-2021-eular.2052

\section{POS0980 \\ ANALYSIS OF PRIMARY CARE CONSULTATION PATTERNS TO AID DIAGNOSIS OF AXIAL SPONDYLOARTHRITIS - AN EXPLORATORY CASE SERIES}

M. Al-Attar ${ }^{1}$, W. J. Gregory ${ }^{1,2}$, J. Mcbeth ${ }^{3,4}$, W. Dixon ${ }^{1,3,4} \cdot{ }^{1}$ Rheumatology Department, Salford Royal NHS Foundation Trust, Salford, United Kingdom ${ }^{2}$ Faculty of Health, Psychology and Social Care, Manchester Metropolitan University, Manchester, United Kingdom; ${ }^{3}$ Centre for Epidemiology Versus Arthritis, University of Manchester, Manchester, United Kingdom; ${ }^{4} \mathrm{NIHR}$ Manchester Musculoskeletal Biomedical Research Centre, Central Manchester University Hospitals NHS Foundation Trust, Manchester, United Kingdom

Background: Patients with Axial Spondyloarthritis (AxSpA) often suffer a significant delay to diagnosis. This is associated with poorer outcomes in quality of life, functional capabilities and work productivity [1]. These patients are frequent consulters to primary care in the years preceding rheumatology referral [2]. We hypothesise that analysis of primary care consultation patterns may identify as-yet undiagnosed disease, and suggest that implementing an automated diagnostic algorithm may support early action in primary care.

Objectives: To undertake a preliminary exploration of primary care consultation patterns in patients with a delayed diagnosis of $\mathrm{AxSpA}$ and identify themes for further research.

Methods: The study was run in Salford, UK, where unique linkage exists across electronic health records (EHR) from primary and secondary care. A dataset of patients with AxSpA was obtained from 2018-2020 hospital physiotherapy clinic records. Ten patients with a time to diagnosis $\geq 5$ years were randomly selected for this exploratory analysis. Diagnostic delay was calculated based on rheumatology clinic letter documentation. Age, sex, and HLA-B27 status were recorded.
All "Problem" codes from the primary care EHR up to the point of diagnosis were manually reviewed.

Results: Age at diagnosis was 32-49 years with seven males and three females. Seven were HLA-B27 positive. The average delay to diagnosis was 15.8 years (range 5-30).

On average, patients had 15 primary care consultations (range 5-24) between first coded AxSpA-related symptom and rheumatology referral. Around half of these codes were potentially AxSpA-related (for example, see Figure 1).

Six patients had a coded history of back pain. Two patients presented with other axial symptoms, including: rib pain, MSK chest pain and sciatica.

Five patients presented with peripheral joint symptoms, including: ankle pain shoulder pain, knee problem, pain in arm, medial epicondylitis elbow, hip pain and groin pain. Of these, four had multiple presentations and three had a previous visit with axial pain

Two patients had uveitis preceding axial symptoms. One patient had periphera joint symptoms (hip pain) preceding uveitis.

Inconsistent codes were used for the same problem presenting at different times in nine cases, including: back pain, backache, low back pain, lower back pain.

Other relevant codes were used in seven cases, including: stiffness, arthritis, saw physiotherapist and referred to pain clinic.

Figure 1 illustrates the consultation pattern for a male patient who first presented to primary care with back pain at the age of 35. Despite a relatively typical presentation, his diagnosis was made incidentally 10 years later after an ESR was checked for unrelated reasons. He was significantly disabled in function at the point of being referred to rheumatology.

Figure 1: Example of a patient's primary care consultation pattern

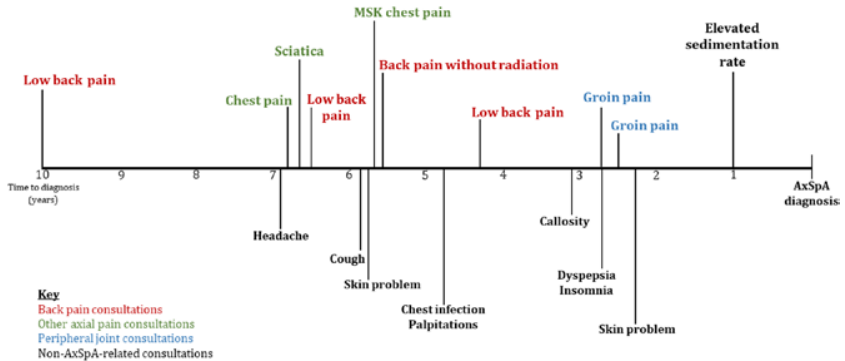

Conclusion: Our preliminary analysis suggests that patients with a delayed diagnosis of AxSpA have repeated primary care visits with potentially recognisable symptoms of their disease. These findings support the feasibility of future automated detection, with areas of focus including recognition of non-back pain axial symptoms, extra-articular manifestations, and peripheral joint symptoms.

Whilst half of presentations were not directly AxSpA-related, modern machine learning techniques have the ability to explore whether the pattern or frequency of these consultations are relevant to identifying undiagnosed disease. Such methods can also highlight patterns obscured by extensive data sets and inconsistent coding, with opportunity for implementation back into primary care.

\section{REFERENCES:}

[1] Redeker I et al. Determinants of diagnostic delay in axial spondyloarthritis: an analysis based on linked claims and patient-reported survey data. Rheumatology (Oxford) 2019;58:1634-8.

[2] Yi E et al. Clinical, Economic, and Humanistic Burden Associated With Delayed Diagnosis of Axial Spondyloarthritis: A Systematic Review. Rheumatol Ther. 2020;7(1):65-87

Disclosure of Interests: None declared.

DOI: 10.1136/annrheumdis-2021-eular.2124 